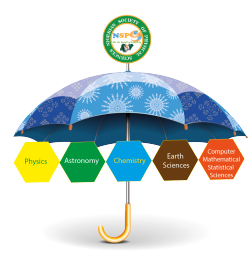

\title{
Numerical Algorithms for Direct Solution of Fourth Order Ordinary Differential Equations
}

\author{
J. O. Kuboye, O. R. Elusakin*, O. F. Quadri \\ Department of Mathematics, Federal University Oye-Ekiti, Oye-Ekiti, Nigeria
}

\begin{abstract}
This paper examines the derivation of hybrid numerical algorithms with step length(k) of five for solving fourth order initial value problems of ordinary differential equations directly. In developing the methods, interpolation and collocation techniques are considered. Approximated power series is used as interpolating polynomial and its fourth derivative as the collocating equation. These equations are solved using Gaussianelimination approach in finding the unknown variables $a_{j}, \mathrm{j}=0, \ldots, 10$ which are substituted into basis function to give continuous implicit scheme. The discrete schemes and its derivatives that form the block are obtained by evaluating continuous implicit scheme at non-interpolating points. The developed methods are of order seven and the results generated when the methods were applied to fourth order initial value problems compared favourably with existing methods.
\end{abstract}

DOI:10.46481/jnsps.2020.100

Keywords: Interpolation, Collocation, Block methods, Fourth order, Ordinary differential equations

Article History :

Received: 07 May 2020

Received in revised form: 10 August 2020

Accepted for publication: 15 August 2020

Published: 01 November 2020

(C)2020 Journal of the Nigerian Society of Physical Sciences. All rights reserved. Communicated by: F. Y. Eguda

\section{Introduction}

The general fourth order initial value problem of ordinary differential equations of the form

$$
\begin{aligned}
& y^{i v}=f\left(x, y(x), y^{\prime}(x), y^{\prime \prime}(x), y^{\prime \prime \prime}(x)\right), \\
& y\left(x_{0}\right)=y_{1}, y^{\prime}\left(x_{0}\right)=y_{2}, y^{\prime \prime}\left(x_{0}\right)=y_{3}
\end{aligned}
$$

is considered in this article. In the past, solving fourth order ordinary differential equations (ODEs) requires reducing

\footnotetext{
${ }^{*}$ Corresponding author tel. no: $+23480 \times x \times x 572$

Email address: opeyemielusakin21@gmail.com (O. R. Elusakin )
}

the differentials to systems of first order ODEs and approximate numerical method for the first order would be used to solve the system. This approach is been attached with lots of setbacks which include: computational burden, lots of human effort, complexity in developing computer code which affects the accuracy of the method in terms of error. This was extensively discussed by researchers like Awoyemi [1], Fatunla [2] and Lambert [3]. Due to several disadvantages found in reduction method, the direct method of solving ODEs of higher order was developed by lots of scholars which include Akeremale et al. [4], Abolarin et al. [5], Kuboye et. al [6], Omar \& Kuboye [7], Adeyefa [8], Abdullahi et al. [9], Adeniyi \& Mohammed [10], Olabode [11], Adesanya et al.[12], Omar \& Suleiman [13] 
and Familua \& Omole [14]. Specifically, numerical methods for solving equation (1) were proposed by Omar and Kuboye[15], Areo and Omole[16] and Mohammed[17]. These current methods solved directly equation (1) but its accuracy in terms of error can still be improved. Therefore, this paper examines the derivation and implementation of the efficient numerical algorithm for solving fourth order ordinary differential equations directly and it focuses on improving the accuracy of the existing methods.

\section{Methodology}

This section considers derivation of block methods for direct solution of fourth order ODEs.

\subsection{Derivation of First Block Method(FBM)}

Power series approximate solution of the form

$$
y(x)=\sum_{j=0}^{k+5} a_{j} x^{j}
$$

is used as interpolating polynomial where $\mathrm{k}=5$. The fourth derivative of equation(2) gives:

$$
y^{i v}(x)=\sum_{j=4}^{k+5} j(j-1)(j-2)(j-3) a_{j} x^{j-4}
$$

Equation (2) is interpolated at $x=x_{n+i}, i=0(1) 2$ and $\frac{5}{2}$ and equation (3) is collocated at $x=x_{n+i}, i=0(1) 5$ and $\frac{5}{2}$. Interpolation and collocation equations are combined together to give a non-linear system of equations of the form:

$$
\begin{aligned}
& \sum_{j=0}^{k+5} a_{j} x_{n+i}^{j}=y_{n+i} \\
& \sum_{j=4}^{k+5} j(j-1)(j-2)(j-3) a_{j} x_{n+i}^{j-4}=f_{n+i}
\end{aligned}
$$

The unknown variables $a_{j}^{\prime} s$ in (4) are gotten with the use of Gaussian elimination approach which are substituted into equation (2) and this yields a continuous implicit scheme of the form

$$
\sum_{j=0}^{k-3} \alpha_{j}(t) y_{n+j}+\alpha_{\frac{5}{2}} y_{n+\frac{5}{2}}=h^{4} \sum_{j=0}^{k} \beta_{j}(t) f_{n+j}+h^{4} \lambda_{\frac{5}{2}} f_{n+\frac{5}{2}}
$$

where $t=\frac{x-x_{n+k-1}}{h}$

$$
\left[\begin{array}{l}
\alpha_{0}(t) \\
\alpha_{1}(t) \\
\alpha_{2}(t) \\
\alpha_{\frac{5}{2}}(t)
\end{array}\right]=\left[\begin{array}{cccc}
\frac{-9}{5} & \frac{-27}{10} & \frac{-13}{10} & \frac{-1}{5} \\
8 & \frac{34}{3} & 5 & \frac{2}{3} \\
-18 & \frac{45}{2} & \frac{17}{2} & 1 \\
\frac{64}{5} & \frac{208}{15} & \frac{24}{5} & \frac{8}{15}
\end{array}\right]\left[\begin{array}{l}
t^{0} \\
t^{1} \\
t^{2} \\
t^{3}
\end{array}\right]
$$


$\left[\begin{array}{l}\beta_{0}(t) \\ \beta_{1}(t) \\ \beta_{2}(t) \\ \beta_{\frac{5}{2}}(t) \\ \beta_{3}(t) \\ \beta_{4}(t) \\ \beta_{5}(t)\end{array}\right]=T\left[\begin{array}{l}t^{0} \\ t^{2} \\ t^{3} \\ t^{5} \\ t^{6} \\ t^{7} \\ t^{8} \\ t^{9} \\ t^{10}\end{array}\right]$

where T $=\left[\begin{array}{ccccccc}\frac{297000}{290304000} \frac{404694}{290304000} & \frac{75279}{290304000} \frac{-102185}{290304000} & \frac{72576}{290304000} \frac{36288}{290304000} & \frac{-2880}{290304000} \frac{-7200}{290304000} & \frac{-2080}{290304000} \frac{-192}{290304000} \\ \frac{3441528}{11612160} \frac{5154498}{11612160} & \frac{2433933}{11612160} \frac{323717}{11612160} & \frac{32256}{11612160} \frac{15232}{11612160} & \frac{-1728}{11612160} \frac{-3072}{11612160} & \frac{-800}{11612160} \frac{-64}{11612160} \\ \frac{6797304}{5806080} \frac{10732194}{5806080} & \frac{5745021}{5806080} \frac{1207573}{5806080} & \frac{-145152}{5806080} \frac{-6048}{5806080} & \frac{10944}{5806080} \frac{12384}{5806080} & \frac{2720}{5806080} \frac{192}{5806080} \\ \frac{560520}{2268000} \frac{690174}{2268000} & \frac{419859}{2268000} \frac{267195}{2268000} & \frac{-129024}{2268000} \frac{-46592}{2268000} & \frac{11520}{2268000} \frac{9600}{2268000} & \frac{1920}{2268000} \frac{128}{2268000} \\ \frac{1716984}{5806080} \frac{3760866}{5806080} & \frac{3494877}{5806080} \frac{153384}{5806080} & \frac{-290304}{5806080} \frac{-72576}{5806080} & \frac{27072}{5806080} \frac{16704}{5806080} & \frac{3040}{5806080} \frac{192}{5806080} \\ \frac{187272}{11612160} \frac{129150}{11612160} & \frac{-353709}{11612160} \frac{-650629}{11612160} & \frac{-169344}{11612160} \frac{-3136}{11612160} & \frac{20160}{11612160} \frac{7392}{11612160} & \frac{1120}{11612160} \frac{64}{11612160} \\ \frac{428760}{290304000} \frac{508266}{290304000} & \frac{-76719}{290304000} \frac{-322779}{290304000} & \frac{290304}{290304000} \frac{266112}{290304000} & \frac{118080}{290304000} \frac{2880}{290304000} & \frac{3680}{290304000} \frac{192}{290304000}\end{array}\right]$

The coefficient of first and higher derivatives of (5) give

$$
\left[\begin{array}{c}
\alpha_{0}^{\prime}(t) \\
\alpha_{1}^{\prime}(t) \\
\alpha_{2}^{\prime}(t) \\
\alpha_{\frac{5}{2}}^{\prime}(t)
\end{array}\right]=\left[\begin{array}{ccc}
\frac{27}{10} & \frac{26}{10} & \frac{-6}{10} \\
\frac{34}{3} & \frac{30}{3} & \frac{6}{3} \\
\frac{45}{2} & \frac{34}{2} & \frac{-6}{2} \\
\frac{208}{15} & \frac{144}{15} & \frac{124}{15}
\end{array}\right] \frac{1}{h}
$$


$\left[\begin{array}{c}\beta_{0}^{\prime} \\ \beta_{1}^{\prime} \\ \beta_{2}^{\prime} \\ \beta_{\frac{5}{2}}^{\prime} \\ \beta_{3}^{\prime}(t) \\ \beta_{4}^{\prime}(t) \\ \beta_{5}^{\prime}(t)\end{array}\right]=S\left[\begin{array}{l}t^{0} \\ t^{1} \\ t^{2} \\ t^{4} \\ t^{5} \\ t^{6} \\ t^{7} \\ t^{8} \\ t^{9}\end{array}\right]$

Where S $=\left[\begin{array}{cccccccccc}\frac{-134898}{96768000} & \frac{-50186}{96768000} & \frac{102185}{96768000} & \frac{-120960}{96768000} & \frac{-72576}{96768000} & \frac{6720}{96768000} & \frac{19200}{96768000} & \frac{6240}{96768000} & \frac{640}{96768000} \\ \frac{5154498}{11612160} & \frac{4867866}{11612160} & \frac{971151}{11612160} & \frac{161280}{11612160} & \frac{91392}{11612160} & \frac{-12096}{11612160} & \frac{-24576}{11612160} & \frac{-7200}{11612160} & \frac{-640}{11612160} \\ \frac{3577398}{1935360} & \frac{3830014}{1935360} & \frac{1207573}{1935360} & \frac{-241920}{1935360} & \frac{-120960}{1935360} & \frac{25536}{1935360} & \frac{33024}{1935360} & \frac{8160}{1935360} & \frac{640}{1935360} \\ \frac{690174}{2268000} & \frac{839718}{2268000} & \frac{801585}{2268000} & \frac{-645120}{2268000} & \frac{-2795520}{2268000} & \frac{80640}{2268000} & \frac{76800}{2268000} & \frac{17280}{2268000} & \frac{1280}{2268000} \\ \frac{1253622}{1935360} & \frac{2329918}{1935360} & \frac{1533845}{1935360} & \frac{-483840}{1935360} & \frac{-145152}{1935360} & \frac{63168}{1935360} & \frac{44544}{1935360} & \frac{9120}{1935360} & \frac{640}{1935360} \\ \frac{-129150}{11612160} & \frac{707418}{11612160} & \frac{1951887}{11612160} & \frac{846720}{11612160} & \frac{18816}{11612160} & \frac{-141120}{11612160} & \frac{-59136}{11612160} & \frac{-10080}{11612160} & \frac{-640}{11612160} \\ \frac{169422}{96768000} & \frac{-51146}{96768000} & \frac{-322775}{96768000} & \frac{4838405}{96768000} & \frac{532224}{96768000} & \frac{275520}{96768000} & \frac{76800}{96768000} & \frac{11040}{96768000} & \frac{640}{96768000}\end{array}\right]$

$$
\left[\begin{array}{c}
\alpha_{0}^{\prime \prime}(t) \\
\alpha_{1}^{\prime \prime}(t) \\
\alpha_{2}^{\prime \prime}(t) \\
\alpha_{\frac{5}{2}}^{\prime \prime}(t)
\end{array}\right]=\left[\begin{array}{cc}
\frac{13}{5 h^{2}} & \frac{-6}{5 h^{2}} \\
\frac{10}{h^{2}} & \frac{4}{h^{2}} \\
\frac{17}{h^{2}} & \frac{-6}{h^{2}} \\
\frac{48}{5 h^{2}} & \frac{16}{5 h^{2}}
\end{array}\right]\left[\begin{array}{l}
t^{0} \\
t^{1}
\end{array}\right]
$$


$\left[\begin{array}{l}\beta_{0}^{\prime \prime}(t) \\ \beta_{1}^{\prime \prime}(t) \\ \beta_{2}^{\prime \prime}(t) \\ \beta_{\frac{5}{2}}^{\prime \prime}(t) \\ \beta_{3}^{\prime \prime}(t) \\ \beta_{4}^{\prime \prime}(t) \\ \beta_{5}^{\prime \prime}(t)\end{array}\right]=U\left[\begin{array}{c}t^{0} \\ t^{1} \\ t^{3} \\ t^{4} \\ t^{5} \\ t^{6} \\ t^{7} \\ t^{8}\end{array}\right]$

where $U=\left[\begin{array}{cccccccc}\frac{-25093}{48384000} & \frac{102185}{48384000} & \frac{-241920}{48384000} & \frac{-181440}{48384000} & \frac{20160}{48384000} & \frac{67200}{48384000} & \frac{24960}{48384000} & \frac{2880}{48384000} \\ \frac{811311}{1935360} & \frac{323717}{1935360} & \frac{107520}{1935360} & \frac{76160}{1935360} & \frac{-12096}{1935360} & \frac{-28672}{1935360} & \frac{-9600}{1935360} & \frac{-960}{1935360} \\ \frac{1915007}{967680} & \frac{1207573}{967680} & \frac{-483840}{967680} & \frac{-302400}{967680} & \frac{76608}{967680} & \frac{11558}{967680} & \frac{32640}{967680} & \frac{2880}{967680} \\ \frac{139953}{378000} & \frac{267195}{378000} & \frac{-430080}{378000} & \frac{-232960}{378000} & \frac{80640}{378000} & \frac{89600}{378000} & \frac{23040}{378000} & \frac{1920}{378000} \\ \frac{1164959}{967680} & \frac{1533845}{967680} & \frac{-967680}{967680} & \frac{-362880}{967680} & \frac{18950}{967680} & \frac{155904}{967680} & \frac{36480}{967680} & \frac{2880}{967680} \\ \frac{117903}{1935360} & \frac{650629}{1935360} & \frac{564480}{1935360} & \frac{15680}{1935360} & \frac{-141120}{1935360} & \frac{-68992}{1935360} & \frac{-13440}{1935360} & \frac{-960}{1935360} \\ \frac{-25573}{48384000} & \frac{-322775}{48384000} & \frac{967680}{48384000} & \frac{1330560}{48384000} & \frac{826560}{48384000} & \frac{268800}{48384000} & \frac{44160}{48384000} & \frac{2880}{48384000}\end{array}\right]$

$\left[\begin{array}{c}\alpha_{0}^{\prime \prime \prime}(t) \\ \alpha_{1}^{\prime \prime \prime}(t) \\ \alpha_{2}^{\prime \prime \prime}(t) \\ \alpha_{\frac{5}{2}}^{\prime \prime \prime}(t)\end{array}\right]=\left[\begin{array}{c}\frac{-6}{5} \\ 4 \\ -6 \\ \frac{16}{5}\end{array}\right] t^{0}$

$\left[\begin{array}{l}\beta_{0}^{\prime \prime \prime}(t) \\ \beta_{1}^{\prime \prime \prime}(t) \\ \beta_{2}^{\prime \prime \prime}(t) \\ \beta_{\frac{5}{2}}^{\prime \prime \prime}(t) \\ \beta_{3}^{\prime \prime \prime}(t) \\ \beta_{4}^{\prime \prime \prime}(t) \\ \beta_{5}^{\prime \prime \prime}(t)\end{array}\right]=V\left[\begin{array}{c}t^{0} \\ t^{2} \\ t^{3} \\ t^{4} \\ t^{5} \\ t^{6} \\ t^{7}\end{array}\right]$ 


$$
\text { where } V=\left[\begin{array}{ccccccc}
\frac{20437}{9676800} & \frac{-14515}{9676800} & \frac{-145152}{9676800} & \frac{20160}{9676800} & \frac{80640}{9676800} & \frac{3494}{9676800} & \frac{4608}{9676800} \\
\frac{323717}{967680} & \frac{322560}{967680} & \frac{304640}{967680} & \frac{-60480}{967680} & \frac{-172032}{967680} & \frac{-67200}{967680} & \frac{-7680}{967680} \\
\frac{1207573}{967680} & \frac{-1451520}{967680} & \frac{-1209600}{967680} & \frac{383040}{967680} & \frac{69350}{967680} & \frac{228480}{967680} & \frac{23040}{967680} \\
\frac{-53439}{75600} & \frac{258048}{75600} & \frac{186368}{75600} & \frac{-80640}{75600} & \frac{-107520}{75600} & \frac{-32256}{75600} & \frac{-3072}{75600} \\
\frac{1533845}{967680} & \frac{-2903040}{967680} & \frac{-1451520}{967680} & \frac{947520}{967680} & \frac{935424}{967680} & \frac{255360}{967680} & \frac{23040}{967680} \\
\frac{650629}{1935360} & \frac{1935360}{1693440} & \frac{62720}{1935360} & \frac{-705600}{1935360} & \frac{-413952}{1935360} & \frac{-94080}{1935360} & \frac{-7680}{1935360} \\
\frac{-64555}{9676800} & \frac{580608}{9676800} & \frac{1064448}{9676800} & \frac{826560}{9676800} & \frac{322560}{9676800} & \frac{61824}{9676800} & \frac{4608}{9676800}
\end{array}\right]
$$

Discrete schemes and its derivatives are derived by evaluating (5) as well as its derivatives at grid points and non-grid points which are used to form the block

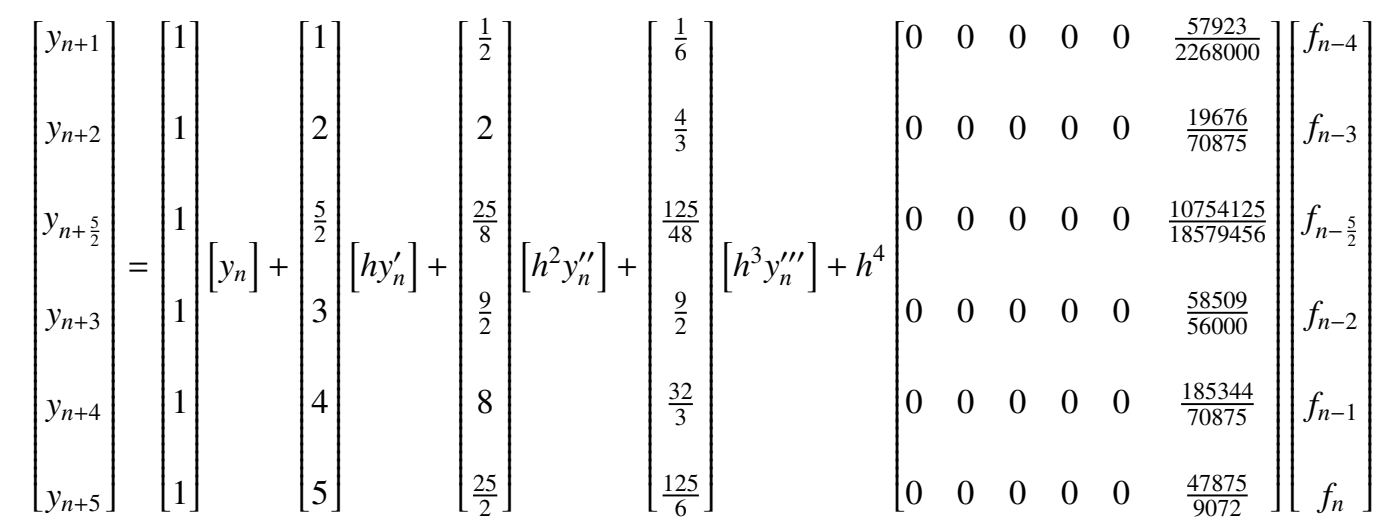

$$
+h^{4}\left[\begin{array}{cccccc}
\frac{413}{10368} & \frac{-8977}{90720} & \frac{9292}{70875} & \frac{-2279}{36288} & \frac{29}{3780} & \frac{-6593}{9072000} \\
\frac{428}{567} & \frac{-122}{81} & \frac{140288}{70875} & \frac{-2672}{2835} & \frac{4}{35} & \frac{764}{70875} \\
\frac{33650625}{18579456} & \frac{-30109375}{9289728} & \frac{89375}{20736} & \frac{-19109375}{9289728} & \frac{171875}{688128} & \frac{-437875}{18579456} \\
\frac{16137}{4480} & \frac{3267}{560} & \frac{6948}{875} & \frac{-243}{64} & \frac{207}{448} & \frac{-4887}{112000} \\
\frac{28928}{2835} & \frac{-7936}{567} & \frac{1441792}{70875} & \frac{-27136}{2835} & \frac{32}{27} & \frac{-7936}{70875} \\
\frac{1609375}{72576} & \frac{-484375}{18144} & \frac{23500}{567} & \frac{-671875}{36288} & \frac{15625}{6048} & \frac{-2375}{10368}
\end{array}\right]\left[\begin{array}{l}
f_{n+1} \\
f_{n+2} \\
f_{n+\frac{5}{2}} \\
f_{n+3} \\
f_{n+4} \\
f_{n+5}
\end{array}\right]
$$

\subsection{Derivation of Second Block Method(SBM)}

Equation(2) is interpolated at $x=x_{n+i}, i=0(1) 2$ and $\frac{9}{4}$ and equation(3) is collocated at $x=x_{n+i}, i=0(1) 5$ and $\frac{9}{4}$. The same steps used in deriving the first block method are also employed and this produces the block method 


$$
\begin{aligned}
& {\left[\begin{array}{l}
y_{n+1} \\
y_{n+2} \\
y_{n+\frac{9}{4}} \\
y_{n+3} \\
y_{n+4} \\
y_{n+5}
\end{array}\right]=\left[y_{n}\right]+\left[\begin{array}{l}
1 \\
\frac{9}{4} \\
3 \\
4 \\
5
\end{array}\right]\left[h y_{n}^{\prime}\right]+\left[\begin{array}{c}
\frac{1}{2} \\
\frac{81}{32} \\
\frac{9}{2} \\
8 \\
\frac{4}{3} \\
\frac{25}{2}
\end{array}\right]\left[h^{2} y_{n}^{\prime \prime}\right]+\left[\begin{array}{c}
\frac{1}{6} \\
\frac{243}{128} \\
\frac{9}{2} \\
\frac{32}{3} \\
\frac{125}{6}
\end{array}\right]\left[h^{3} y_{n}^{\prime \prime \prime}\right]+h^{4}\left[\begin{array}{cccccc}
0 & 0 & 0 & 0 & 0 & \frac{69043}{2721600} \\
0 & 0 & 0 & 0 & 0 & \frac{11696}{42525} \\
0 & 0 & 0 & 0 & 0 & \frac{1191908439}{2936012800} \\
0 & 0 & 0 & 0 & 0 & \frac{5793}{5600} \\
0 & 0 & 0 & 0 & 0 & \frac{22016}{8505} \\
0 & 0 & 0 & 0 & 0 & \frac{568625}{108864}
\end{array}\right]\left[\begin{array}{l}
f_{n-4} \\
f_{n-\frac{9}{4}} \\
f_{n-3} \\
f_{n-2} \\
f_{n-1}
\end{array}\right]} \\
& +h^{4}\left[\begin{array}{cccccc}
\frac{76921}{1814400} & \frac{-1139}{6480} & \frac{594688}{3274425} & \frac{-6749}{181440} & \frac{7421}{1270080} & \frac{-11717}{19958400} \\
\frac{11248}{14175} & \frac{-7558}{2835} & \frac{8978432}{3274425} & \frac{-1576}{2835} & \frac{344}{3969} & \frac{-1352}{155925} \\
\frac{3705501897}{2936012800} & \frac{-169057287}{41943040} & \frac{229379121}{55193600} & \frac{-247763043}{293601280} & \frac{540947889}{4110417920} & \frac{-425211849}{32296140800} \\
\frac{84159}{22400} & \frac{-2349}{224} & \frac{148224}{13475} & \frac{-5031}{2240} & \frac{1377}{3920} & \frac{-8667}{246400} \\
\frac{150272}{14175} & \frac{-10496}{405} & \frac{8388608}{297675} & \frac{-15872}{2835} & \frac{17888}{19845} & \frac{-256}{2835} \\
\frac{1668125}{72576} & \frac{-115625}{2268} & \frac{7520000}{130977} & \frac{-378125}{36288} & \frac{509375}{254016} & \frac{-147625}{798336}
\end{array}\right]\left[\begin{array}{l}
f_{n+1} \\
f_{n+2} \\
f_{n+\frac{9}{4}} \\
f_{n+3} \\
f_{n+4} \\
f_{n+5}
\end{array}\right]\left[\begin{array}{l} 
\\
\vdots
\end{array}\right]
\end{aligned}
$$

\section{Analysis of the method}

Properties of the methods are examined in this section.

\subsection{Order of block methods}

In finding the order of the block methods, the method proposed by Lambert[3] is employed whereby Taylor series expansion are used in expanding the $\mathrm{y}$ and f-functions and by further comparing the coefficients of h,this gives the block methods to be of order $[7,7,7,7,7,7]^{T}$.

\subsection{Zero-stability}

A linear multi-step method is said to be zero-stable if the roots $r_{s}, \mathrm{~s}=1,2, \ldots, \mathrm{N}$ (grid and non grid points) of the first characteristics polynomial defined by $p(r)=\operatorname{det}\left(r A^{\prime}-B^{\prime}\right)$ satisfy $\left|r_{s}\right|<1$ and the root $|r|=1$ having multiplicity not exceeding one.(Lambert [3])

$$
\text { Where } A^{\prime}=\left[\begin{array}{llllll}
1 & 0 & 0 & 0 & 0 & 0 \\
0 & 1 & 0 & 0 & 0 & 0 \\
0 & 0 & 1 & 0 & 0 & 0 \\
0 & 0 & 0 & 1 & 0 & 0 \\
0 & 0 & 0 & 0 & 1 & 0 \\
0 & 0 & 0 & 0 & 0 & 1
\end{array}\right]
$$




$$
B^{\prime}=\left[\begin{array}{llllll}
0 & 0 & 0 & 0 & 0 & 1 \\
0 & 0 & 0 & 0 & 0 & 1 \\
0 & 0 & 0 & 0 & 0 & 1 \\
0 & 0 & 0 & 0 & 0 & 1 \\
0 & 0 & 0 & 0 & 0 & 1 \\
0 & 0 & 0 & 0 & 0 & 1
\end{array}\right]
$$

Therefore $r=0,0,0,0,0,1$. Hence the zero-stability of first block method is confirmed which is also applied to the second block method.

\subsection{Convergence}

According to Awoyemi[1], Equation(5) converges if it is zero-stable and consistent.This implies that the developed methods converged.

\section{Test Problems}

The following fourth order initial value problems[I.V.P] are solved in order to examine the accuracy of the methods

Problem 1:

$y^{i v}=x, y(0)=0, y^{\prime}(0)=1, y^{\prime \prime}(0)=y^{\prime \prime \prime}=0, h=0.1$

Exactsolution: $y(x)=\frac{x^{5}}{120}+x$

Source: Mohammed[17]

Problem 2:

$y^{i v}-y=0, y(0)=1, y^{\prime}(0)=0, y^{\prime \prime}(0)=-2, y^{\prime \prime \prime}(0)=0, h=\frac{1}{320}$

Exactsolution: $y(x)=\frac{-1}{4} e^{x}-\frac{1}{4} e^{-x}+\frac{3}{2} \cos (x)$

Source: Areo and Omole[16]

Problem 3:

$y^{i v}=\left(y^{\prime}\right)^{2}-y y^{\prime \prime \prime}-4 x^{2}+e^{x}\left(1-4 x+x^{2}\right), \quad y(0)=1, y^{\prime}(0)=1, y^{\prime \prime}(0)=3, y^{\prime \prime \prime}(0)=1, h=\frac{0.1}{32}$

Exactsolution: $y(x)=x^{2}+e^{x}$

Source: Familua and Omole [14]

The following acronyms are used in the Tables below

ES - Exact Solution

CS - Computed Solution

FBM - First Block Method 
SBM - Second Block Method

EIM[17] - Error in Mohammed[17]

EIAO[16]- Error in Areo and Omole[16]

EIOK [15]- Error in Omar and Kuboye[15]

EIFBM - Error in First Block Method

EISBM - Error in Second Block Method

EIFO [14] - Error in Familua and Omole [14]

Table 1. ES and CS of FBM for Problem 1

\begin{tabular}{|c|c|c|}
\hline $\mathrm{x}$ & ES & CS \\
\hline 0.1 & 0.100000083333333340 & 0.100000083333333340 \\
\hline 0.2 & 0.200002666666666690 & 0.200002666666666690 \\
\hline 0.3 & 0.300020250000000040 & 0.300020250000000040 \\
\hline 0.4 & 0.400085333333333350 & 0.400085333333333400 \\
\hline 0.5 & 0.500260416666666650 & 0.500260416666666760 \\
\hline 0.6 & 0.600647999999999960 & 0.600648000000000070 \\
\hline 0.7 & 0.701400583333333330 & 0.701400583333333550 \\
\hline 0.8 & 0.802730666666666700 & 0.802730666666666700 \\
\hline 0.9 & 0.904920750000000050 & 0.904920750000000160 \\
\hline 1.0 & 1.008333333333333300 & 1.008333333333333500 \\
\hline
\end{tabular}

Table 2. ES and CS of SBM for Problem 1

\begin{tabular}{|c|c|c|}
\hline $\mathrm{x}$ & $\mathrm{ES}$ & $\mathrm{CS}$ \\
\hline 0.1 & 0.100000083333333340 & 0.100000083333333340 \\
\hline 0.2 & 0.200002666666666690 & 0.200002666666666690 \\
\hline 0.3 & 0.300020250000000040 & 0.300020250000000100 \\
\hline 0.4 & 0.400085333333333350 & 0.400085333333333400 \\
\hline 0.5 & 0.500260416666666650 & 0.500260416666666650 \\
\hline 0.6 & 0.600647999999999960 & 0.600648000000000070 \\
\hline 0.7 & 0.701400583333333330 & 0.701400583333333220 \\
\hline 0.8 & 0.802730666666666700 & 0.802730666666666810 \\
\hline 0.9 & 0.904920750000000050 & 0.904920750000000160 \\
\hline 1.0 & 1.008333333333333300 & 1.008333333333333300 \\
\hline
\end{tabular}

\section{Discussion of Results}

In Tables 1 and 2, exact and computed solutions of FBM and SBM for solving problem 1 are shown. Table 3 reveals the efficiency of these block methods (EISBM and EISBM) as compared favourably with EIM[17] and EIOK[15]. Furthermore, exact and computed solutions of the newly developed block

Table 3. Comparison of EIFBM and EISBM with EIM[17] and EIOK[15] for solving problem 1

\begin{tabular}{|c|c|c|c|c|}
\hline $\mathrm{x}$ & EIFBM & EISBM & $\mathrm{EIM}(2010)$ & $\mathrm{EIOK}(2016)$ \\
\hline 0.1 & $0.0000000 \mathrm{e}+00$ & $0.0000000 \mathrm{e}+00$ & $7.000024 \mathrm{E}-10$ & $1.002087 \mathrm{E}-12$ \\
\hline 0.2 & $0.0000000 \mathrm{e}+00$ & $0.0000000 \mathrm{e}+00$ & $8.9999912-10$ & $0.000000 \mathrm{E}+00$ \\
\hline 0.3 & $0.0000000 \mathrm{e}+00$ & $5.5511151 \mathrm{e}-17$ & $2.599993 \mathrm{E}-09$ & $0.000000 \mathrm{E}+00$ \\
\hline 0.4 & $5.5511151 \mathrm{e}-17$ & $5.5511151 \mathrm{e}-17$ & $5.100033 \mathrm{E}-09$ & $0.000000 \mathrm{E}+00$ \\
\hline 0.5 & $1.1102230 \mathrm{e}-16$ & $0.0000000 \mathrm{e}+00$ & $7.799979 \mathrm{E}-09$ & $1.002087 \mathrm{E}-12$ \\
\hline 0.6 & $1.1102230 \mathrm{e}-16$ & $1.1102230 \mathrm{e}-16$ & $1.180009 \mathrm{E}-08$ & $2.755907 \mathrm{E}-12$ \\
\hline 0.7 & $2.2204460 \mathrm{e}-16$ & $1.1102230 \mathrm{e}-16$ & $1.180009 \mathrm{E}-08$ & $3.507306 \mathrm{E}-12$ \\
\hline 0.8 & $0.0000000 \mathrm{e}+00$ & $1.1102230 \mathrm{e}-16$ & $1.410006 \mathrm{E}-08$ & $3.507306 \mathrm{E}-12$ \\
\hline 0.9 & $1.1102230 \mathrm{e}-16$ & $1.1102230 \mathrm{e}-16$ & $1.880000 \mathrm{E}-08$ & $4.175549 \mathrm{E}-12$ \\
\hline 1.0 & $2.2204460 \mathrm{e}-16$ & $0.0000000 \mathrm{e}+00$ & $1.008335 \mathrm{E}-08$ & $4.759970 \mathrm{E}-12$ \\
\hline
\end{tabular}

Table 4. ES and CS of FBM for Problem 2

\begin{tabular}{|c|c|c|}
\hline $\mathrm{x}$ & $\mathrm{ES}$ & $\mathrm{CS}$ \\
\hline 0.0031250 & 1.000009765628973500 & 1.000009765628973700 \\
\hline 0.0062500 & 1.000039062563578400 & 1.000039062563578400 \\
\hline 0.0093750 & 1.000087890946866900 & 1.000087890946867100 \\
\hline 0.0125000 & 1.000156251017263000 & 1.000156251017263500 \\
\hline 0.0156250 & 1.000244143108567400 & 1.000244143108567400 \\
\hline 0.0187500 & 1.000351567649961900 & 1.000351567649962400 \\
\hline 0.0218750 & 1.000478525166021100 & 1.000478525166021300 \\
\hline 0.0250000 & 1.000625016276719800 & 1.000625016276719600 \\
\hline 0.0281250 & 1.000791041697446400 & 1.000791041697446800 \\
\hline 0.0312500 & 1.000976602239017000 & 1.000976602239017000 \\
\hline
\end{tabular}

Table 5. ES and CS of SBM for Problem 2

\begin{tabular}{|c|c|c|}
\hline $\mathrm{x}$ & $\mathrm{ES}$ & $\mathrm{CS}$ \\
\hline 0.0031250 & 1.000009765628973500 & 1.000009765628973900 \\
\hline 0.0062500 & 1.000039062563578400 & 1.000039062563578400 \\
\hline 0.0093750 & 1.000087890946866900 & 1.000087890946866900 \\
\hline 0.0125000 & 1.000156251017263000 & 1.000156251017263500 \\
\hline 0.0156250 & 1.000244143108567400 & 1.000244143108567100 \\
\hline 0.0187500 & 1.000351567649961900 & 1.000351567649962100 \\
\hline 0.0218750 & 1.000478525166021100 & 1.000478525166021100 \\
\hline 0.0250000 & 1.000625016276719800 & 1.000625016276719600 \\
\hline 0.0281250 & 1.000791041697446400 & 1.000791041697445900 \\
\hline 0.0312500 & 1.000976602239017000 & 1.000976602239017200 \\
\hline
\end{tabular}

Table 6. Comparison of EIFBM and EISBM with EIAO[16] for solving problem 2

\begin{tabular}{|c|c|c|c|}
\hline $\mathrm{x}$ & EIFBM & EISBM & EIAO (2015) \\
\hline 0.0031250 & $2.2204460 \mathrm{e}-016$ & $4.4408921 \mathrm{e}-016$ & $4.440892 \mathrm{e}-16$ \\
\hline 0.0062500 & $0.0000000 \mathrm{e}+000$ & $0.0000000 \mathrm{e}+000$ & $2.176037 \mathrm{e}-14$ \\
\hline 0.0093750 & $2.2204460 \mathrm{e}-016$ & $0.0000000 \mathrm{e}+000$ & $.771916 \mathrm{e}-13$ \\
\hline 0.0125000 & $4.4408921 \mathrm{e}-016$ & $4.4408921 \mathrm{e}-016$ & $7.666090 \mathrm{e}-13$ \\
\hline 0.0156250 & $0.0000000 \mathrm{e}+000$ & $2.2204460 \mathrm{e}-016$ & $2.367773 \mathrm{e}-12$ \\
\hline 0.0187500 & $4.4408921 \mathrm{e}-016$ & $2.2204460 \mathrm{e}-016$ & $5.932477 \mathrm{e}-12$ \\
\hline 0.0218750 & $2.2204460 \mathrm{e}-016$ & $0.0000000 \mathrm{e}+000$ & $1.287681 \mathrm{e}-11$ \\
\hline 0.0250000 & $2.2204460 \mathrm{e}-016$ & $2.2204460 \mathrm{e}-016$ & $2.517841 \mathrm{e}-11$ \\
\hline 0.0281250 & $4.4408921 \mathrm{e}-016$ & $4.4408921 \mathrm{e}-016$ & $4.546752 \mathrm{e}-11$ \\
\hline 0.0312500 & $0.0000000 \mathrm{e}+000$ & $2.2204460 \mathrm{e}-016$ & $7.712331 \mathrm{e}-11$ \\
\hline
\end{tabular}

\begin{tabular}{|c|c|c|}
\multicolumn{3}{c}{ Table 7. ES and CS of FBM for Problem 3 } \\
\begin{tabular}{|c|c|c|}
\hline $\mathrm{x}$ & ES & CS \\
\hline 0.103125 & 1.119264744787591900 & 1.119264744969084200 \\
\hline 0.206250 & 1.271599493198048500 & 1.271599504741302500 \\
\hline 0.306250 & 1.452110907065013100 & 1.452111029006491400 \\
\hline 0.406250 & 1.666216862500122800 & 1.666217515460942200 \\
\hline 0.506250 & 1.915347109920916500 & 1.915349507140536000 \\
\hline 0.603125 & 2.191581593606204900 & 2.191588302867649500 \\
\hline 0.703125 & 2.514440293333696500 & 2.514456732090109900 \\
\hline 0.803125 & 2.877516387746607200 & 2.877551937602963200 \\
\hline 0.903125 & 3.282936158805099100 & 3.283006004031709900 \\
\hline 1.003125 & 3.733049511495175400 & 3.733176679391747100 \\
\hline
\end{tabular}
\end{tabular}


Table 8. ES and CS of SBM for Problem 3

\begin{tabular}{|c|c|c|}
\hline $\mathrm{x}$ & ES & $\mathrm{CS}$ \\
\hline 0.103125 & 1.119264744787591900 & 1.119264744966372600 \\
\hline 0.206250 & 1.271599493198048500 & 1.271599504536039800 \\
\hline 0.306250 & 1.452110907065013100 & 1.452111026692671300 \\
\hline 0.406250 & 1.666216862500122800 & 1.666217502634746300 \\
\hline 0.506250 & 1.915347109920916500 & 1.915349459022614800 \\
\hline 0.603125 & 2.191581593606204900 & 2.191588166231517800 \\
\hline 0.703125 & 2.514440293333696500 & 2.514456393591375100 \\
\hline 0.803125 & 2.877516387746607200 & 2.880551715825508700 \\
\hline 0.903125 & 3.282936158805099100 & 3.283004543615038400 \\
\hline 1.003125 & 3.733049511495175400 & 3.733174005515217600 \\
\hline
\end{tabular}

Table 9. Comparison of EIFBM and EISBM with EIFO [14] for solving problem 3

\begin{tabular}{|c|c|c|c|}
\hline $\mathrm{x}$ & EIFBM & EISBM & EIFO[14] \\
\hline 0.103125 & $1.8149238 \mathrm{e}-010$ & $1.7878077 \mathrm{e}-010$ & $9.02145880 \mathrm{e}-10$ \\
\hline 0.206250 & $1.1543254 \mathrm{e}-008$ & $1.1337991 \mathrm{e}-008$ & $1.216821428 \mathrm{e}-09$ \\
\hline 0.306250 & $1.2194148 \mathrm{e}-007$ & $1.1962766 \mathrm{e}-007$ & $1.21681228 \mathrm{e}-09$ \\
\hline 0.406250 & $6.5296082 \mathrm{e}-007$ & $6.4013462 \mathrm{e}-007$ & $1.713796095 \mathrm{e}-09$ \\
\hline 0.506250 & $2.3972196 \mathrm{e}-006$ & $2.3491017 \mathrm{e}-006$ & $1.481970916 \mathrm{e}-08$ \\
\hline 0.603125 & $6.7092614 \mathrm{e}-006$ & $6.5726253 \mathrm{e}-006$ & $3.058338503 \mathrm{e}-08$ \\
\hline 0.703125 & $1.6438756 \mathrm{e}-005$ & $1.6100258 \mathrm{e}-005$ & $4.941858156 \mathrm{e}-08$ \\
\hline 0.803125 & $3.5549856 \mathrm{e}-005$ & $3.5007632 \mathrm{e}-005$ & $7.128679089 \mathrm{e}-08$ \\
\hline 0.903125 & $6.9845227 \mathrm{e}-005$ & $6.8384810 \mathrm{e}-005$ & $1.058773080 \mathrm{e}-07$ \\
\hline 1.003125 & $1.2716790 \mathrm{e}-004$ & $1.2449402 \mathrm{e}-004$ & $1.445520074 \mathrm{e}-07$ \\
\hline
\end{tabular}

methods for the solution of problems 2 and 3 are demonstrated in Tables 4, 5, 7 and 8. These methods outperform method proposed by Areo and Omole [16] in terms of accuracy. In addition, the performance of these methods in solving problem 3 is not encouraging as the accuracy is lower when the comparison is made with EIFO [14]. However, the capability of these methods in solving the nonlinear equation is established in Table 9. Finally, it is evident in Tables 3, 6 and 9 that SBM is better than FBM in solving fourth order ODEs.

\section{Conclusion}

In this paper, new numerical algorithms for solving fourth order initial value problems of ODEs via multistep collocation approach were developed. The use of approximated power series as a basis function and its fourth derivatives as collocating equation were considered. The derived methods are efficient in the solution of fourth order ODEs as depicted in Tables 3, 6 and 9. The accuracy of these numerical models is found better compared with some of the existing methods in terms of error. Hence, FBM and SBM are viable numerical methods for solving fourth order initial value problems.

\section{Acknowledgments}

We thank the referees and editor for the creative comments in making improvements to this paper.
References

[1] D. A. Awoyemi, "Class of continuous methods for general second order initial value problems in ordinary differential equations", International Journal of Computer Mathematics 72 (2009) 29.

[2] S. O. Fatunla, Numerical methods for initial value problems in ordinary differential equations, Academic press Inc. Harcourt Brace Jovanovich Publishers, New York. (1988)

[3] J. D. Lambert, Computational method in ordinary differential equation, John Wiley and Sons, Inc. (1973)

[4] O. C. Akeremale, J. O. Kuboye, S. H. Yeak, E. A. Abununyi \& S. Olaiju, " Hybrid-block numerical method for solving second order ordinary differential equations", International Journal of Computational Analysis 3 (2020) 25.

[5] O. E. Abolarin, E. O. Adeyefa, J. O. Kuboye \& B. G. Ogunware, "A novel multi derivative hybrid method for numerical treatment of higher order ordinary differential equations", Al Dar Research Journal of Sustainability 4 (2020) 43.

[6] J. O. Kuboye, Z. Omar, O. E. Abolarin \& R. Abdelrahim, "Generalized hybrid block method for solving second order ordinary differential equations directly", Research and Reports on Mathematics 2 (2018) 2.

[7] Z. Omar \& J. O. Kuboye, "Comparison of block methods with different step-lengths for solving second order ordinary differential equations", Journal of Computational and Theoretical Nanoscience Science and Technology 15 (2018) 966.

[8] E.O. Adeyefa, "Orthogonal-based hybrid block method for solving general second order initial value problems," Italian journal of pure and applied mathematics 37 (2017) 659.

[9] Y. A. Abdullahi, Z. Omar \& J. O. Kuboye, "Derivation of block predictor-block corrector method for direct solution of third order ordinary differential equations", Global Journal of Pure and Applied Mathematics 12 (2016) 342 .

[10] U. Mohammed \& R.B. Adeniyi, "Derivation of five -step block hybrid backward differential formulas (HBDF) through the continuous multistep collocation for solving second order differential equation", Pacific Journal of Science and Technology 15 (2014) 89.

[11] B. T. Olabode, "An accurate scheme by block method for the third order ordinary differential equation", Pacific journal of science and technology 10 (2009) 136

[12] A. O. Adesanya, M. O. Udoh \& A. M. Ajileye, "A new hybrid block method for direct solution of general third order initial value problems of ordinary differential equations", International Journal of Pure and Applied Mathematics 4 (2013) 42.

[13] Z. Omar \& M. Suleiman, "Solving higher order odes directly using parallel 2-point explicit block method", Matematika. Pengintegrasian Teknologi Dalam Sains Matematik. Universiti Sains Malaysia 21 (2005) 15 .

[14] A. B. Familua \& E. O. Omole, "Five points mono hybrid linear multistep method for solving nth order ordinary differential equations using power series function", Asian Research Journal of Mathematics (Science Domain International) 3 (2017) 1.

[15] J. O. Kuboye \& Z. Omar, "New Zero-stable block method for direct solution of fourth order ordinary differential equation", Indian Journal of Science and Technology 8 (2015) 1.

[16] E. A. Areo \& E. O. Omole, "Half-Step symmetric continuous hybrids block method for the numerical solutions of fourth order ordinary differential equations", Archives of Applied Science Research 7 (2015) 39.

[17] U. Mohammed, "A six step block method for solution of fourth order ordinary differential equations", The Pacific Journal of Science and Technology 11 (2010) 259. 